\begin{tabular}{ll}
$\begin{array}{l}\text { Abstract } 013 \text { Table } 1 \\
\text { pregnancy outcomes }\end{array}$ & Description of the cohort at first visit and \\
\hline Median maternal age at conception (IQR) & 31.9 years (29-35) \\
Median disease duration at the time of pregnancy (IQR) & 10 years (6-14) \\
Median duration from the last flare (IQR) & 4.1 years (2-6) \\
Mean duration of pregnancy (IQR) & 37.1 weeks (36-40) \\
Birthweight median value (IQR) & 2802.1 grams (2595-3231) \\
Patients with active disease at the beginning of & $5(6.6 \%)$ \\
pregnancy (\%) & \\
LLDAS BUT NOT remission (\%) & $5(6.6 \%)$ \\
LLDAS (\%) & $60(80 \%)$ \\
Complete remission off treatment (\%) & $5(6.6 \%)$ \\
Clinical remission off treatment (\%) & $10(13.3 \%)$ \\
Clinical remission on treatment (\%) & $21(28 \%)$ \\
Complete remission on treatment (\%) & $17(22.6 \%)$ \\
Preterm prelabour rupture of membranes & $11(14.6 \%)$ \\
Preterm delivery (\%) & $19(25.3 \%)$ \\
SGA infant (\%) & $14(18.6 \%)$ \\
IUGR (\%) & $1(1.3 \%)$ \\
IUFD (\%) & $2(2.6 \%)$ \\
Gestational diabetes (\%) & $4(5.3 \%)$ \\
\hline
\end{tabular}

pregnancy with respect to active patients (OR 12.47, $\mathrm{p}<0.02$ ). Patients in LLDAS but not remission showed an increased risk of flare with respect to remitted patients $(p<0.001)$ while no significant differences were found between patients in clinical versus complete remission. No associations were found between disease status at the beginning of pregnancy and obstetric complications.

Conclusions These data confirm that disease remission is one important predictor of pregnancy outcomes in SLE. While serology seems not to have a substantial role, a residual disease activity at conception might impact on pregnancy outcomes. Available definitions of remission and LLDAS could be valid treatment targets in the family planning perspective.

\section{THE INFLUENCE OF ANTIPHOSPHOLIPID-ANTIBODIES ON INR VALUES MEASURED WITH THE COAGUCHEK}

Chong Ying Wong, Albert Huisman, Rolf Urbanus, Maarten Limper. Rheumatology and Clinical Immunology, University Medical Center Utrecht, Utrecht, The Netherlands

\subsection{6/lupus-2020-eurolupus.26}

Introduction Patients with the antiphospholipid syndrome on anticoagulant therapy can be monitored with the INR using Point Of Care (POC) devices. However, INR values could be falsely elevated due to antiphospholipid-antibodies using POC devices. Previous studies that have compared INR values measured with POC devices and a laboratory coagulometer in APS patients varied in the methods, which makes the interpretation of the results difficult. We have, therefore, conducted this single center study to investigate whether INR values in APS patients measured with the most commonly used POC device (CoaguChek) in the Netherlands are comparable with whole blood INR values in our laboratory in the UMC Utrecht.

Methods INR values measured by the CoaguChek and by the coagulometer in our laboratory measured with the Owren method using a rabbit brain derived thromboplastin were compared in 20 consecutive APS patients. A paired students T-test was performed to compare both means and a $\mathrm{p}$ value $<0,05$ was considered as statistically significant. Linear regression analysis was performed to express the correlation between both test methods. A Bland-Altman plot was constructed to illustrate the agreement of the POC and laboratory INR. A difference $>0,5$ was considered clinically significant.

Results No statistically significant difference between the INR measured with the CoaguChek and the coagulometer in our laboratory was found ( $p>0,05)$. Furthermore, an acceptable correlation was found in the linear regression analysis $(\mathrm{r}=0,893)$. However, in 3 patients with triple antiphospholipid antibody positivity, a clinically relevant difference in the INR was found $(>0,5)$.

Conclusion In conclusion, the CoaguChek generates comparable results with the coagulometer in our clinic. However, in a subset of patients with triple positivity the CoaguChek show a clinical relevant difference. Therefore, results of the CoaguChek in this group of patients should be interpreted with caution.

\section{CONCORDANCE BETWEEN THE NEW SLE-DAS, DORIS AND DORIA REMISSION CRITERIA FOR SLE: ARE THEY DIFFERENT IN A REAL-LIFE CLINICAL SETTING?}

${ }^{1}$ Helena Assunção, ${ }^{2,3}$ Diogo Jesus, ${ }^{1,3}$ Luís Inês. ${ }^{1}$ Rheumatology, Centro Hospitalar Universitário de Coimbra, Coimbra; ${ }^{2}$ Rheumatology, Centro Hospitalar de Leiria, Leiria; ${ }^{3}$ Faculty of Health Sciences, University of Beira Interior, Covilhã, Portugal

\subsection{6/lupus-2020-eurolupus.27}

Background Systemic Lupus Erythematosus Disease Activity Score (SLE-DAS), a new, continuous measure, presents improved sensitivity to change as compared to SLE Disease Activity Index (SLEDAI-2K). It comprises 17 items, including important manifestations absent in SLEDAI (hemolytic anemia, gastrointestinal and cardiopulmonary involvement). The Doria and DORIS clinical remission criteria are both based in SLEDAI. This study aims to compare the attainment of clinical remission defined by SLE-DAS, DORIS and Doria criteria in a real-life clinical setting.

Methods Cross-sectional study of all SLE patients fulfilling ACR'97 and/or SLICC'12 classification criteria followed at an academic lupus clinic from January to September 2019. Fulfillment of DORIS, Doria and SLE-DAS clinical remission status was verified for each patient. The SLE-DAS clinical remission criteria were defined as a score of 0 in all clinical items of SLE-DAS and prednisone dose of $0-5 \mathrm{mg} / \mathrm{day}$. We compared the attainment of clinical remission for each patient according to the three definitions. Sensitivity, specificity, positive and negative predictive values of SLE-DAS remission for Doria and for DORIS remission were calculated.

Results The study population included 293 patients (female = $86.7 \%$; mean age $=47.7$ years; mean disease duration $=14.4$ years). The proportion of patients in clinical remission was $76.5 \%$ as defined by the DORIS and Doria criteria. Patients in clinical remission according to the SLE-DAS definition exactly matched those defined by either Doria or DORIS criteria and there were no discordant cases. From patients in clinical remission, 17\%, 93.3\%, and 30.4\% were treated with 